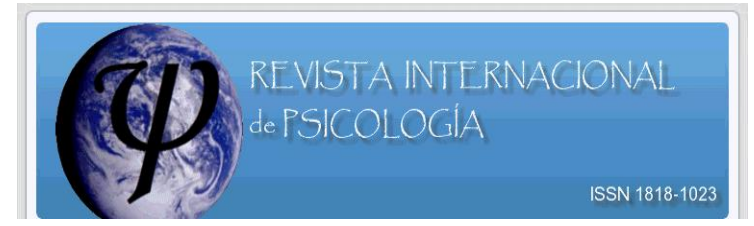

\title{
ESTILOS COGNITIVOS, DESARROLLO OPERATORIO Y PRECONCEPCIONES
}

\author{
Mikel Aramburu Oyarbide ${ }^{1}$
}

\section{Introducción}

La enseñanza tradicional se ha caracterizado por dar prioridad a la acumulación de contenidos conceptuales en la mente de los alumnos. De lo que se trataba era de que el estudiante asimilara de una forma receptiva y pasiva estructuras conceptuales previamente organizadas. Fueron Piaget y Vygotskii los pioneros en entender el aprendizaje como un proceso de construcción en el que los significados emergen en la interacción del individuo con el medio. Desde este punto de vista, el proceso curricular tratará de conectar con el proceso de construcción del conocimiento del alumno. Si la enseñanza tradicional se centraba en la enseñanza de la red conceptual de cada disciplina, la enseñanza por descubrimiento trató de apoyarse en los procesos mentales y en las actividades del alumno. La enseñanza constructivista tratará de fundamentarse tanto en los procesos psicológicos de los alumnos como en la red conceptual de las disciplinas, es decir, tratará de lograr tanto una significatividad lógica como una significatividad psicológica.

La significatividad lógica se refiere a que los contenidos deben tener una estructura interna y una coherencia lógica. La significatividad psicológica requiere que el contenido que se presenta al alumno tenga una relación no arbitraria con sus conocimientos previos. Es decir, que los nuevos conocimientos deben de insertarse en la red de conocimientos previamente construidos. La significatividad lógica hace referencia a la estructuración lógica del contenido. La significatividad psicológica hace referencia a la integración de los nuevos conocimientos en la estructura de conocimientos previos del alumno. Por supuesto que esta doble significatividad no sería suficiente si el alumno no estuviera motivado para aprender, estableciendo relaciones entre los nuevos conocimientos y sus conocimientos previos. En el aprendizaje significativo los conocimientos nuevos se relacionan con los anteriores. Conceptos clave de la red de conocimientos previos harán de puentes cognitivos que funcionarán como organizadores de la nueva red.

\footnotetext{
${ }^{1}$ Universidad del País Vasco. Correo electrónico: mikel@ yahoo.com
} 
El debate relacionado con los fines de la educación sigue en pie. Para unos, el objetivo primario de la educación es conducir a la persona a los niveles que corresponden a sus potenciales. Para otros, el objetivo es asimilar contenidos conceptuales, procedimentales y actitudinales. Para los primeros, lo importante es lograr la competencia cognitiva general y llegar a los niveles de desarrollo universales; resultan baldíos los esfuerzos por enseñar destrezas específicas. Para los segundos, no está claro que los estadios del desarrollo infantil sean universales, ya que si fuera así los individuos alcanzarían los niveles evolutivos superiores sin la necesidad de ayudas específicas por parte de los educadores; para éstos, el objetivo de la educación no es fomentar el desarrollo natural, sino potenciar el máximo nivel de adquisiciones. Sin decantarnos a favor de los unos o de los otros, hemos de decir que no existen ni procesos evolutivos puros ni procesos de aprendizaje puros. Aun admitiendo que las capacidades cognitivas generales pudieran ser universales, habría diferencias significativas a la hora de su aplicación a la resolución de problemas concretos. Es decir, algunas potencialidades del desarrollo de la persona no se podrían actualizar sin la ayuda de un Currículo educativo que programe los aprendizajes específicos.

\section{Desarrollo del Pensamiento Lógico y aprendizaje signiticativo}

El desarrollo del Pensamiento Lógico del niño ha de atravesar unas etapas, antes de llegar al estadio del Pensamiento Formal. El niño que se halla en el estadio del Pensamiento Concreto es incapaz de transferir automáticamente las competencias que ha adquirido en un campo a otro campo de aplicación. En el estadio de las operaciones formales, las competencias debieran de ser en principio independientes del ámbito en el que se han adquirido. En la práctica, sin embargo, lo que se ha adquirido en un contexto no se transfiere automáticamente a otros contextos.

En el nivel del Pensamiento Formal "lo real" está supeditado a "lo posible". "Lo real" no deja de ser una de las actualizaciones de lo que es posible. El mundo real no es más que el subconjunto de "lo que es" dentro del campo de "lo que podría" o de "lo que pudo ser". El Pensamiento Formal se basa en representaciones proposicionales, centrándose en la estructura 
formal, más que en el contenido, en la estructura de conjunto, más que en las situaciones específicas. La posición de Piaget se basó en que el Pensamiento Formal era una condición necesaria y suficiente para llegar al conocimiento científico. Muchos seguidores suyos trataron de aplicar estas ideas a la enseñanza de las ciencias, razonando de la siguiente forma: si el desarrollo del Pensamiento Formal se da independientemente de los contenidos concretos de aprendizaje, bastaría con enseñar las estructuras del Pensamiento Formal, para que los estudiantes fueran capaces de entender cualquier contenido científico. La enseñanza de contenidos específicos dejó de ser un objetivo, para pasar a ser un medio para el logro del nivel del Pensamiento Formal. Se trata de que los alumnos aprendan el método científico, más que los conceptos fundamentales de la ciencia.

Superada la euforia de los primeros descubrimientos de Piaget, se han llevado a cabo múltiples investigaciones, empezando por el mismo Piaget, y se ha llegado a la conclusión de que el nivel del Pensamiento Formal no es universal, ni siquiera entre los alumnos universitarios. Además, se ha descubierto que los esquemas del nivel formal no se adquieren todos al mismo tiempo, descubrimiento que desbarata la opinión de que el Pensamiento Formal tiene una estructura de conjunto. Se observa, además, que en la resolución de las tareas formales, aparte de la estructura lógica de la tarea, es relevante el contenido de la misma, y que la comprensión de este contenido está condicionada por la existencia de las concepciones previas del alumno.

Piaget y colaboradores encontraron en los Estilos Cognitivos y en las representaciones de los niños las razones por las que un gran número de sujetos no llegue al Nivel Formal en el nivel de actuación. En la resolución de las tareas formales interfieren los Estilos Cognitivos, las Concepciones Espontáneas de los sujetos y el contenido de la tarea. Con la intención de dar una salida al problema del desfase entre la competencia y la actuación del sujeto, se ha hablado de los Esquemas Operatorios Formales, que serían unas estructuras intermedias, con un nivel de especificidad mayor que las estructuras del Pensamiento Formal. El nivel de desarrollo de los Esquemas Operatorios Formales, juntamente con los Estilos Cognitivos y los conocimientos previos de los alumnos condicionarán los efectos que puedan tener las experiencias educativas en el alumno. Es 
necesario establecer la pertinente diferencia entre lo que el alumno puede aprender por sí mismo, sin ayuda externa, y lo que puede aprender con la ayuda de otra persona, para situar la Zona de Desarrollo Potencial en la que ha de actuar la labor educativa. Es esa zona que media entre el desarrollo efectivo y el desarrollo potencial la que debemos tratar de cubrir con el desarrollo del Currículum Educativo. El nivel de desarrollo efectivo condiciona los aprendizajes posibles, en cuanto que la significatividad de éstos depende de su fundamentación en el nivel adquirido. Si el alumno adquiere contenidos sin relacionarlos con su idiosincrasia, valores, objetivos, actitudes y sentimientos, se tratará de un aprendizaje externo, sin significatividad alguna. El alumno aprende un contenido (sea conceptual, procedimental o actitudinal) cuando consigue darle un significado. La construcción del significado es el proceso clave del aprendizaje. El aprendizaje significativo es el que surge de la experiencia personal, aquel en el que los contenidos nuevos se integran en la red de conocimientos previos, en el que el alumno decide lo que ha de aprender, el que tiene en cuenta los aspectos afectivos y actitudinales, y aquel que se basa en la autoevaluación continua. Si queremos que el aprendizaje del alumno no sea meramente memorístico, habremos de lograr que tenga un nivel mínimo de significatividad.

\section{Aprendizaje y concepciones espontáneas (preconcepciones) de los niños}

Llamamos concepciones espontáneas a aquellas que el niño tiene sobre un tema antes de escuchar las explicaciones del profesor. Surgen en la mente del niño en su interacción con el medio, sin ninguna influencia especial de la enseñanza. Son personales y pueden incluso ser inducidas, a través por ejemplo de la influencia de la lengua. Es habitual que los individuos pertenecientes a un grupo compartan algunas de las concepciones o preconcepciones, sean éstas erróneas o no, ya que dependen del contexto en el que surgen. La influencia del contexto y de la lengua es manifiesta en estas preconcepciones.

Estas preconcepciones, a pesar de que a menudo son científicamente incorrectas, suelen ser predictoras y eficaces. Suelen tener un nivel de abstracción limitado y se limitan a lo que es perceptible. Son intuitivas y se desvían a menudo de las concepciones científicas. Suelen tener una mínima estructura teorética y resultan difíciles de cambiar. 
Muchos estudiantes universitarios siguen manteniendo sus preconcepciones erróneas, ya que para cambiarlas no basta con provocar contradicciones con respecto a conceptos aislados. Hace falta cambiar la estructura de la teoría implícita. Muchas de la preconcepciones de los alumnos reproducen conceptos de la Historia del Conocimiento Científico. Utilizan un tipo de razonamiento lineal y cumplen una función de adaptación práctica, permitiendo al individuo enfrentarse al mundo a través de un filtro de expectativas. Son estructuras teóricas mínimas que nos dan una sensación de control sobre los cambios que se dan en el medio. Funcionan como mecanismos de resistencia ante los cambios sorpresivos.

Para cambiar las preconcepciones erróneas del alumno hay que activar las ideas del mismo. Hay que provocar un conflicto entre las ideas erróneas y las nuevas, de modo que éstas puedan sustituir a aquellas. Se trata de reestructurar la estructura conceptual, y no de cambiar unos conceptos por otros. Pero no se trata de una reestructuración cognitiva global, sino de cambios limitados a ámbitos cognitivos concretos, ya que la ciencia no es el camino para el desarrollo de facultades globales, sino para la adquisición de conocimientos específicos. El nivel de Pensamiento Formal es una condición necesaria, pero no suficiente, para aprender la ciencia. El no haber logrado el nivel de Pensamiento Formal puede impedir a los alumnos la comprensión de los conceptos científicos. Es por ello que los profesores se esmeran en que los alumnos logren el nivel operatorio formal. Pero, hay además otros factores, tal como hemos subrayado más arriba, que inciden en la comprensión del alumno: el Estilo Cognitivo y las Preconcepciones Espontáneas.

\section{Estudio de las preconcepciones del Área de Naturaleza}

He utilizado el método clínico-descriptivo al inicio del estudio de las ideas previas de los alumnos. Me he valido de un método similar al utilizado por Piaget: he descrito las ideas previas de los alumnos. Después de esa fase descriptiva, en un segundo momento, me he sumergido en una investigación cuantificada de las concepciones infantiles, correlacionándolas con tests estandarizados de Estilos Cognitivos y del Desarrollo Operatorio del Pensamiento Lógico. En la medida en que me interesaba conocer las 
concepciones naturales de los alumnos sobre los temas del Área de Naturaleza, he llevado a cabo un análisis sistemático de las mismas. Con ese objetivo, he seleccionado los bloques temáticos que se enseñan en ese área y he analizado las preconcepciones infantiles más sobresalientes en cada área.

He aquí los principales bloques temáticos:

- El ser humano, los animales y las plantas.

- La composición de la materia.

- Transformaciones y conservación de la materia.

- Fuerza y movimiento. Universo.

Se muestran a continuación los resultados del estudio descriptivo sobre las concepciones espontáneas:

\section{Elser humano, los animales y las plantas}

He aquí una relación sucinta de algunas de las preconcepciones infantiles:

- Lo que diferencia a la materia orgánica de la inorgánica es la composición atómica de cada una de ellas. Los átomos de los seres vivientes están vivos, están en "marcha", sus moléculas están vivas. Las características macroscópicas de los seres vivientes se dan también en su nivel microscópico. De la misma forma que hay seres vivientes y no vivientes, hay también átomos vivientes y no vivientes.

- En el aparato circulatorio la sangre no se percibe como transportador de alimentos nutritivos; es la misma sangre la que es nutriente, surgida de los alimentos y compuesta por ellos. Entienden que la sangre está compuesta de alimentos y oxígeno. Incluso en el medio cultural está bastante extendida esa idea. Parece ser que el significado de los términos científicos cambia cuando pasa por el filtro del sentido común. 
- Entienden la fotosíntesis como parte de la respiración. Los niños dicen que las plantas hacen la fotosíntesis de día y respiran de noche.

- No conocen la función de la fecundación ni en los animales ni en las plantas. Confunden el espermatozoide y la semilla, de la misma forma que confunden el polen y la semilla.

\section{Preconcepciones sobre la composición de la materia}

- Para muchos niños las células no tienen átomos. Las piedras sí, tienen átomos. Pero las piedras no están compuestas sólo de átomos, sino de algo más consistente y duro. De la misma forma que consideran que entre las partículas de aire hay aire, los niños consideran también que entre los átomos de la piedra hay una sustancia dura.

- Los niños consideran al átomo como la unidad fundamental de la materia inorgánica, y a la célula como la unidad fundamental de la materia orgánica.

- No hay necesidad de ningún tipo de mediador entre el ojo y el objeto visto por aquel. En la visión se da un movimiento del ojo hacia el objeto. No tienen idea del reflejo de la luz. Incluso para los que afirman que la luz nos viene por los rayos, el rayo mismo no es luz. El rayo transporta luz.

Los niños tienen una idea continua del aire, de la materia y de la luz: entre los átomos de piedra hay una sustancia dura; entre las partículas de aire hay aire; entre las partículas de luz, hay luz. Son unas preconcepciones erróneas que responden a una visión continua de la materia.

\section{Concepciones sobre la conservación y la transformación de la materia}

A los niños les resulta difícil admitir que la materia se conserva, más allá de sus transformaciones físicas. Piensan que al evaporarse el agua, sus partículas se deshacen, que el agua se convierte en aire, que el agua suelta el oxígeno, y que deja de ser agua. Consideran que cuando el vapor se condensa, el vapor se convierte en agua, pero que no es agua en su forma vaporosa. El cambio de estado es un cambio de sustancia para los niños. 
Los niños consideran que las transformaciones se dan tanto en el nivel macroscópico como en el nivel microscópico. Tienen una concepción continua de la materia. No admiten la concepción discontinua (compuesta por partículas) de la sustancia. Les resulta dificultoso concebir que detrás de las transformaciones la materia se conserva. En las transformaciones físicas, la materia no se conserva para los niños. Menos aún en las transformaciones químicas.

\section{Las concepciones de los niños sobre la fuerza yel movimiento}

Los capítulos dedicados a la fuerza y al movimiento son importantes dentro de la física. Los autores que se han dedicado a estudiar las concepciones infantiles han dedicado bastantes esfuerzos a los mismos. Lo que más sobresale de entre las concepciones sobre estos temas es la referente a que los niños no pueden percibir un movimiento sin una fuerza que le acompañe. Carecen de idea de la inercia. Si un objeto está en movimiento, es debido a un motor que lo ha puesto en movimiento. De la misma forma que para Aristóteles, también para los niños el estado natural de los objetos es el estado de reposo. Para los chinos era el movimiento el estado natural de todos los objetos del universo, y para parar el movimiento hace falta una fuerza opositora. Para los occidentales, sin embargo, por lo menos hasta la teoría de la inercia de Newton, lo que hace que un objeto siga moviéndose es la fuerza y el empuje. En la vida diaria los niños no consideran el rozamiento como fuerza. De ahí que el movimiento continuo implique la existencia de una fuerza continua.

\section{Preconcepciones referentes alcalor}

- El calor es una sustancia para muchos niños.

- Los objetos grandes guardan más calor.

- Los objetos que hay en un aula suelen estar a diferentes temperaturas. Unos materiales son cálidos en sí mismos, y otros son fríos. La temperatura de diferentes objetos metidos en un horno no subirá al mismo nivel: la temperatura de unos materiales subirá más que la temperatura de otros. La temperatura de unas sustancias quedará por debajo del nivel térmico; la de otras sustancias subirá por encima del nivel térmico. 
Estas preconcepciones infantiles están basadas en la experiencia perceptiva. Los niños no conciben la idea de la transferencia e interacción entre objetos y sistemas. El calor es una sustancia que tienen una fuerza motriz y que no está relacionada con la transferencia de la energía entre dos cuerpos. El niño no entiende la temperatura como el nivel de agitación de las partículas. Para entender lo referente a la agitación de las partículas debería de tener una concepción discreta de la materia, y comprender que la materia está compuesta de partículas. Sin la idea de vacío no se entiende la idea de temperatura.

Los niños entienden el calor como una sustancia. Es una concepción paralela a la teoría del calórico de la Historia de la Ciencia. El calórico es un fluido indestructible que al transferirse de un cuerpo a otro hace que descienda la temperatura del primero y que suba la del segundo. Es una sustancia que impregna toda la materia, pero que sólo sale a relucir cuando un cuerpo pierde el equilibrio térmico y el calórico tiene que emigrar de un cuerpo a otro. Existe también una teoría similar, la teoría del flogisto. El flogisto es una sustancia que está presente en todos los cuerpos combustibles. Cuando se quema el cuerpo el flogisto se escapa. Tanto la teoría del calórico como la del flogisto son teorías sustancialistas, muy unidas a lo que percibimos por los sentidos.

\section{Resumen de la teorías previas de los niños}

Los niños de entre 10 y 14 años tienen una creencia bastante generalizada de que los fenómenos del nivel microscópico son similares a lo que ven que ocurre en el nivel macroscópico. Si una sustancia se quema al nivel macroscópico, las partículas microscópicas de la sustancia también se queman. Si una sustancia se evapora o se derrite a nivel microscópico, sus partículas se deshacen a nivel microscópico.

Constatamos una especie de preformismo en los niños. Son muchos los niños que no ven la necesidad de la idea de la fecundación para la procreación. El nuevo ser no deriva de la combinación de la información discreta del óvulo y del espermatozoide.

No comprenden la especificidad de la alimentación de las plantas: no relacionan la fotosíntesis con la alimentación, sino con la respiración. 
Desconocen la realidad de los microorganismos. Identifican a los vivientes con una serie de propiedades perceptibles. No tienen conciencia de seres vivientes microscópicos. En consecuencia, surgen teorías similares a la generación espontánea para explicar fenómenos como la putrefacción, la fermentación etc.

Los niños tienen una concepción continua de la materia. Aun admitiendo las partículas de luz, entre esas partículas sigue habiendo luz, y no vacío. Entre los átomos de piedra, sigue habiendo piedra, y entre las partículas de aire sigue habiendo aire. En lugar de admitir la idea de discontinuidad y de vacío, persisten en la idea de que entre las partículas hay una sustancia aglutinadora que une a esas partículas.

En las transformaciones físicas y químicas la materia no se conserva. En los cambios de estado y en la combustión la sustancia se destruye y desaparece. Las transformaciones macroscópicas suponen transformaciones microscópicas.

No conciben la idea de inercia. Todos los movimientos suponen una fuerza motora.

\section{Los libros de texto, los profesores ylas preconcepciones infantiles}

Los libros de texto a menudo no tienen en cuenta las preconcepciones de los alumnos. En algunos casos, incluso han consolidado algunas concepciones erróneas. Y los profesores tampoco tenemos en cuenta hasta qué punto esas preconcepciones limitan la comprensión de los alumnos. Después de todo, el alumno tomará conciencia de sus concepciones erróneas a través de los textos que maneja y de las explicaciones de sus profesores. Serán éstos quienes harán surgir el conflicto entre las concepciones erróneas y las científicas.

Al programar la secuenciación de los contenidos del Proyecto Curricular, el profesor utilizará, entre otros criterios, su conocimiento de las concepciones erróneas de los alumnos. Basándose en las mismas, estructurará los ejes de las unidades didácticas. Será interesante que incluso el título de la unidad didáctica responda a algún conocimiento 
previo del alumno, de forma que pueda activarla y anclar así sus explicaciones en las ideas previas de los alumnos.

\section{Estilos cognitivos}

Los estilos cognitivos son una especie de filtros de control que tratan de modular los deseos del sujeto de forma que se adecuen a las exigencias de la realidad. En la satisfacción de sus necesidades los rasgos cognitivos del sujeto cumplen una función reguladora importante. La percepción de las personas no dependerá pues únicamente de sus necesidades, sino que estará también regulada por su estructura cognitiva. La función de los controles cognitivos reside en tratar de evitar que las necesidades desorganicen demasiado la percepción del sujeto.

Aparecen dos tendencias cuando se trata de definir los estilos cognitivos:

- La de quienes definen los estilos no sólo como expresión del funcionamiento cognitivo del sujeto, sino como aspectos de la personalidad relacionados con dimensiones no cognitivas de la personalidad.

- La de quienes definen los estilos como preferencias procedimentales de organización perceptiva y categorización conceptual. Es decir, como procedimientos particulares para percibir, pensar, recordar y resolver problemas.

El estilo cognitivo más estudiado ha sido el de la Dependencia-Independencia de Campo. Los Independientes de Campo son los sujetos que perciben la información de forma analítica y sin influencias del medio. Los Dependientes de Campo perciben la información de forma sintética y global. Se distinguen por su forma de integrar las fuentes de percepción de la verticalidad. Mientras que los Independientes de Campo se valen de las sensaciones vestibulares y cenestésicas para definir la orientación vertical, los Dependientes de Campo utilizan los ejes verticales y horizontales del campo visual como marco de referencia para establecer la verticalidad. Fue Witkin quien logró diferenciar a los individuos según las referencias que se utilizan para el establecimiento de la verticalidad: 
los sujetos que confían en el marco visual y los que se valen de la sensación de gravedad. Entre las experiencias que hizo para ello se pueden mencionar las siguientes:

- BAT (Test de ajuste corporal): se le hace sentar al sujeto en una silla situada en una habitación que se puede inclinar hacia la derecha y hacia la izquierda. La silla también se puede inclinar, independientemente de la inclinación de la habitación. Se le sienta al sujeto en la silla previamente inclinada, silla que se encuentra en la habitación que también está inclinada, y se le pide que encuentre la vertical. Hay sujetos que ante esta orden hacen caso omiso de las sensaciones corporales y enderezan su silla tomando como referencia la habitación inclinada tal como aparece en el campo visual. Otros sujetos, sin embargo, utilizan la sensación corporal de gravedad como punto de referencia principal de percepción de la verticalidad.

- RFT (Testa de la barra y del marco): se le sienta al sujeto en una habitación oscura y se le muestra un marco iluminado provisto de una barra giratoria, barra que se puede inclinar independientemente del marco. La tarea del sujeto consiste en llevar a la vertical la barra que previamente estaba inclinada, mientras se mantiene el marco en posición inclinada. Los sujetos que realizan esta tarea se diferencian entre los que buscan la vertical tomando como referencia los estímulos provenientes del campo visual, y los que buscan la vertical tomando como referencia sus sensaciones cenestésicas y gravitatorias.

- RRT (Test de la Habitación Giratoria): se trata de una habitación giratoria en la que se sitúa una silla que se puede inclinar a derecha o a izquierda. Se sienta al sujeto en una silla inclinada y se hace que gire la habitación en su carril circular. Se le pide al sujeto que busque la vertical. Ante esta tarea se diferencian los sujetos que enderezan el cuerpo según la habitación vertical que observan en su campo visual de aquellos sujetos que buscan la vertical según las fuerzas alteradas (fuerza de la gravedad que actúa desde dentro y fuerza centrífuga que actúa desde el exterior) que actúan sobre su cuerpo. 
Los sujetos que en los tres tests toman como punto de referencia el contexto exterior son Dependientes de Campo. Los sujetos que toman como punto de referencia la sensaciones corporales son Independientes de Campo.

\section{EFT (Test de Figuras Enmascaradas)}

Las diferencias constatadas en la percepción de la verticalidad se muestran también en otros ámbitos. Así, en el EFT se le presentan al sujeto dos figuras: una simple y otra compleja, y se le dice que busque la primera en la segunda. Los sujetos que en esta tarea encuentran dificultades para identificar la figura simple dentro de la compleja son las mismas que en los tests anteriores han encontrado dificultades para desentenderse de los estímulos distorsionadores externos: son los sujetos Dependientes de Campo. En la resolución de los problemas que requieren diferenciar una determinada parte extrayéndola del contexto global, los Dependientes de campo encuentran dificultades para separar esos elementos del contexto original y resituarlos en un contexto nuevo.

El Estilo Cognitivo Reflexividad-Impulsividad ha sido también bastante estudiado. Los sujetos Impulsivos son aquellos que actúan de forma inmediata y reflexionan sobre lo que han hecho después de haber actuado. Los sujetos Reflexivos son los que evalúan las diferentes alternativas de respuesta antes de actuar; reflexionan antes de actuar. Si queremos saber si un sujeto es impulsivo o reflexivo, hemos de situarle ante una situación que tiene muchas soluciones diferentes. Es ante las tareas difíciles donde podremos diferenciar a los unos de los otros. Los sujetos impulsivos serán a menudo víctimas de la ansiedad que les lleva a dar una respuesta no meditada. Los sujetos reflexivos son los que se toman el suficiente tiempo para sopesar las diferentes alternativas. La posibilidad de errar en la respuesta les lleva a ser cautos: prefieren demorar la respuesta y dar una respuesta certera a dar una respuesta rápida y errónea.

En cuanto a la orientación profesional de los sujetos de diferentes estilos cognitivos, parece ser que los Independientes de Campo muestran preferencia por los estudios técnico-científicos, mientras que los Dependientes de Campo optarían por las actividades y ciencias de orientación social. En los alumnos Dependientes de campo 
influyen más las opiniones de sus profesores, mientras que los Independientes de campo se rigen por su propio criterio. Los Dependientes de Campo actúan más por motivación externa, y los Independientes de Campo más por motivación interna. Aunque no se pueda decir que un estilo sea mejor que otro, es cierto que los sujetos Independientes de Campo y Reflexivos se adaptan mejor a nuestro sistema escolar. En determinados campos específicos (relaciones sociales, literatura...) y en momentos determinados del proceso de aprendizaje, puede ocurrir que la Dependencia de Campo y la Impulsividad sean consideradas como mejores.

\section{Relación entre los Estilos, las preconcepciones y el desarrollo operatorio}

Siendo nuestra intención el analizar las causas por las que un alumno no llega al estadio Formal del Pensamiento Lógico, hemos hecho por una parte un estudio clínicodescriptivo de las Preconcepciones de los alumnos, y por otra parte, nos hemos introducido en un análisis teórico del Desarrollo Operatorio y de los Estilos Cognitivos. Por fin, intentando cerrar el bucle, hemos hecho un análisis cuantitativo del Desarrollo Operatorio, del estilo Cognitivo y de las Preconcepciones de 133 sujetos del Tercer Ciclo de Primaria y del Primer Ciclo de Secundaria Obligatoria, y hemos llegado a las siguientes conclusiones:

1. En los resultados recogidos tanto a partir del método descriptivo como a partir del método cuantificado ha quedado claro que muchas de las preconcepciones infantiles son persistentes, y que perduran incluso cuando el alumno avanza en edad y promociona de curso en curso. Aunque es cierto que alguna de las preconcepciones erróneas desaparece en la medida en que el alumno progresa a lo largo de los cuatro cursos que hemos estudiado, hay preconcepciones que se solidifican. Esta constatación habla claramente de la dificultad con la que se encuentran los profesores en su labor educativa.

2. Esas preconcepciones aparecen a menudo como muestras de teorías implícitas y tácitas. Resulta posible construir estas teorías soterradas a partir de las concepciones que muestran los alumnos. El hecho de que las preconcepciones puedan estar estructuradas en 
teorías, así como la constatación de que ni los alumnos ni los profesores son conscientes de esas teorías, dificulta el cambio educativo que se pretende.

3. Muchas de las preconcepciones están muy extendidas entre los alumnos. El 50\% de los alumnos del último ciclo de Primaria y del Primer Ciclo de Secundaria Obligatoria comparte las preconcepciones erróneas sobre muchos de los temas objeto de nuestros cuestionarios. Los cuestionarios se han basado en preconcepciones erróneas previamente detectadas por el método descriptivo. El método cuantitativo ha confirmado la constatación de que esas preconcepciones están muy extendidas. Son muy pocas las preconcepciones erróneas que están poco extendidas.

4. La mayor parte de esas preconcepciones son científicamente incorrectas, a pesar de que a menudo predicen bien los acontecimientos. Resultan de utilidad práctica en la medida en que predicen exactamente ciertos acontecimientos. De ahí que los alumnos las mantengan, a pesar de que estén en contradicción con las teorías que reciben en el colegio. Muchas explicaciones científicas son contraintuitivas, y no se generalizan puesto que las teorías erróneas son más intuitivas y contagiosas.

5. En el inicio de la investigación predijimos que los Independientes de Campo mostrarían menos preconcepciones erróneas que los Dependientes de Campo. Los resultados muestran que los Independientes de Campo tienen significativamente menos preconcepciones erróneas que los Dependientes de Campo. Lo cual significa que los Independientes de Campo muestran mayor independencia con respecto a las concepciones previas que se encuentran extendidas en el medio. Hay sin embargo algunas preconcepciones erróneas a las que se adhieren más los Independientes de Campo. Ello puede ser debido a su tendencia a centrarse más en sus convicciones íntimas y en sus teorías personales. La tendencia de los Independientes de Campo a independizarse del medio y a centrarse en sus convicciones son dos tendencias que pueden llevar al sujeto, ya sea a desligarse de las concepciones erróneas que están extendidas, ya sea a centrarse más en sus convicciones erróneas, por mucho que otros no sean partícipes de las mismas. 
Según el estadístico de la regresión, se puede considerar que las preconcepciones infantiles son las variables explicadoras fundamentales de la Independencia de Campo. Se daría una especie de paralelismo entre la capacidad de identificar y discriminar una imagen simple en una imagen compleja y la capacidad de funcionar independientemente con respecto a las teorías implícitas extendidas en el medio.

6. En consonancia con las investigaciones de Pascual-Leone, ha aparecido en nuestra investigación una correlación positiva significativa entre la Independencia de Campo y el Desarrollo del Pensamiento Lógico. Según avanzan en el Desarrollo del Pensamiento Lógico los alumnos aparecen como más independientes de campo, o dicho de otra manera, según van superando la dependencia perceptiva con respecto al medio, van avanzando en el Desarrollo del Pensamiento Lógico. Lo que predecía Pascual-Leone lo demuestra nuestra investigación: lo Independientes de Campo logran mejores resultados en las pruebas piagetianas que los Dependientes de Campo. Los Independientes de Campo logran significativamente mejores resultados que los Dependientes de Campo en las pruebas piagetianas de Peso, Volumen, Disociaciones, Permutaciones, Probabilidades, Péndulo y Curvas Mecánicas. Así, los Estilos Cognitivos resultan ser una herramienta útil a la hora de explicar el fracaso de ciertos alumnos en la realización de las tareas formales. Debido entre otros factores al Estilo Dependencia/Independencia de Campo, la competencia operatoria de los sujetos no se traduce en la correspondiente actuación. De ahí que tengamos que tener en cuenta el Estilo Cognitivo cuando se trata de traducir la competencia en actuación.

7. Se ha encontrado una correlación positiva significativa entre el Desarrollo del Pensamiento Lógico y el rendimiento en el área de Naturaleza. En la medida en que avanza en el Desarrollo del Pensamiento Lógico el alumno rinde mejor en el área de Naturaleza. Según el estadístico de la regresión múltiple, la causa más importante del rendimiento en esa área es el Desarrollo del Pensamiento Lógico. Pero una investigación realizada con una muestra más extensa muestra que el rendimiento en el área de Naturaleza se explica por el nivel de inteligencia, por el rendimiento en el área de Matemáticas y por las Preconcepciones. Tenemos que tener pues en cuenta, que aun siendo manifiesta la correlación entre el 
rendimiento en el área de Naturaleza y el Desarrollo del Pensamiento Lógico, no se pueden olvidar factores como la Inteligencia o las Preconcepciones.

8. Aparece una correlación positiva significativa entre el rendimiento del área de Naturaleza y la Independencia de Campo. El Desarrollo del Pensamiento Lógico es un factor importante para la comprensión de los conceptos del área de Naturaleza, pero también el Estilo Cognitivo resulta de gran importancia: los Independientes de Campo ocupan su espacio mental con esquemas que son importantes para la resolución de problemas, en tanto que los Dependientes de Campo lo ocupan con aspectos que pueden resultar engañosos y les impiden aprovechar todo el espacio.

9. Aparece correlación positiva entre el Desarrollo del Pensamiento Lógico y el hecho de tener menos concepciones erróneas, en los bloques temáticos referentes al Aire, la Tierra, la Reproducción y los Animales. La hipótesis en la que predecimos que los sujetos con más desarrollo del Pensamiento Lógico deberán de mostrar menos concepciones erróneas resulta confirmada en relación con un gran número de bloques temáticos. Asimismo, la hipótesis que dice que los individuos con menos concepciones erróneas han de mostrar un mayor desarrollo del Pensamiento Lógico resulta confirmada. Según la regresión múltiple, serán las preconcepciones infantiles y el desarrollo del área de Matemáticas los que expliquen el desarrollo del Pensamiento Lógico. 


\section{BIBLIOGRAFÍA}

AEBLI,H.(1951): Application à la didactique de la psychologie de Jean Piaget. Neuchâtel, Delachaux et Niestlé. Traducción al castellano: Federico F. Monjardin: Didáctica psicológica. Buenos Aires, Kapelusz, 1973.

ANTUNEZ, S.; DEL CARMEN,L.M.; IMBERNON,F.; PARCERISA,A. y ZABALA,A. (1992) : Del proyecto educativo a la Programación de Aula. Barcelona, Graó.

ASTOLFI J.P. y DEVELAY M. (1991): La didactique des sciences. Paris, P.U.F., Que sais-je?.

AUSUBEL,D.P.; NOVAK,J.D. y HANESIAN,H. (1978): Educational Psychology. A cognitive view. New York, Holt, Rinehart \& Winston. Traducción al castellano: Psicología educativa. Un punto de vista cognoscitivo. México, Trillas, 1983.

BASTIEN,C.(1987): Schèmes et stratégies dans l'activité cognitive de l'enfant. Paris, P.U.F..

BASURKO,F.(1986): Bases Psico-Pedagógicas para la planificación del Ciclo Inicial de las Enseñanzas Medias Tesis doctoral. F.I.C.E. UPV. Pedagogia Saila. Hezkuntzaren teoria eta Historiaren Departamentua.

CAINZOS,M. (1987): Desarrollo y aprendizaje en el Ciclo Superior. Tesis dosctoral. F.I.C.E.- U.P.V. Pedagogia Saila.

CARRETERO,M. y PALACIOS,J.: Los estilos cognitivos. Introducción al problema de las diferencias individuales, Infancia y aprendizaje: 17, 21-28,1982.

CARRETERO,M.: El desarrollo del estilo cognitivo dependencia-independencia de campo, Infancia y aprendizaje, 18, 65-82, 1982.

CARRETERO, M. (1985): Las operaciones concretas. J.Palacios; A.Marchesi y M.Carretero, Psicología evolutiva 2. Desarrollo cognitivo y social en el niño. Madrid, Alianza.

CARRETERO,M. (1985): Las teorías neopiagetianas. Marchesi,A.; Carretero,M. y Palacios,J., Psicología evolutiva 1. Teorías y métodos. Madrid, Alianza.

CARRETERO y colaboradores (1986): Psicología evolutiva 3. Adolescencia, madurez y senectud. Madrid, Alianza. 
CARRETERO,M. (1986): El desarrollo cognitivo en la adolescencia y la juventud: Las operaciones formales. Carretero,M.; Palacios,J. y Marchesi,A., Psicología evolutiva 3. Adolescencia, madurez y senectud. Madrid, Alianza.

CARRetero, M. (1989): Psicología evolutiva 1. Teorías y métodos. Madrid, Alianza.

COLL,C. (1983): Psicología genética y aprendizajes escolares, Madrid, S.XXI.

COLL,C. (1986): Psicología y curriculum. Una aproximación psicológica al curriculum escolar. Barcelona, Cuadernos de Pedagogía. Laia.

COLL,C.(1990): Aprendizaje escolar y construcción del conocimiento. Barcelona, Paidos.

DREVILLON,J. (1980): Pratiques educatives et développement de la pensée opératoire. Paris, P.U.F.

DRIVER,R., GUESNE E., TIBERGHIEN,A. (1985): Childrens ideas en science. Milton Keynes, Open University Press. Traducción al castellano: Pablo Manzano: Ideas Científicas en la infancia y la adolescencia. Madrid, Morata, 1989.

ECHEITA SARRIONANDIA,G. (1988): El mundo adulto en la mente de los niños. La comprensión infantil de las relaciones de intercambio. Madrid, Centro de Publicaciones, C.I.D.E..

ENTWISTLE,N.(1981): Styles of learning and teaching. New York, John Wiley.

FLAVELL,J.H.(1979): La psicología evolutiva de Piaget. Ed. Paidos, Buenos Aires. M.T. Traducción al castellano: Cevasco. The developmental psychology of Jean Piaget. N. Jersey (U.S.A.), Van Nostrand Company Inc. Princeton.

GARCIA RAMOS, J.M. (1989): Los estilos cognitivos y su medida: estudios sobre la dimensión dependencia-independencia de campo, C.I.D.E, Madrid.

GIL,D.; CARRASCOSA,J.; FURIO C. y MTNEZ-TORREGROSA,J. (1991): La enseñanza de las ciencias en la educación secundaria, Barcelona, Horsori, ICE.

GINSBURG (1981): Piaget and Education: the contributions and limits of Genetic Epistemology, New Directions in Piagetian Theory and Practice, Edited by Irving E. Sigel, David M. Brodzinsky, Robert M. Golinkoff, Hillsdale, New Jersey, L.E.A., 315-330 o.

GIORDAN,A. y DE VECCHI,G.(1987): Les origines du savoir (des conceptions des apprenants aux concepts scientifiques). Paris, Delachaux \& Niestlé S.A..Neuchâtel 
(Switzerland). Traducción al castellano: Los orígenes del saber (De las concepciones personales a los conceptos científicos). Sevilla, Diada, 1988.

HALBWACHS,F. (1974): La pensée physique chez l'enfant et le savant, Newchâtel (Switzerland), Delachaux et Niestlé.

HARLEN W.(1985): Teaching and learning primary science, Paul Chapman Publishing Ltd. Traducción al castellano: Enseñanza y aprendizaje de las ciencias, Madrid, Morata, 1989.

HEZKUNTZA, UNIBERTSITATE ETA IKERKETA SAILA (1992): Oinarrizko

Curriculum Diseinua. Derrigorrezko Bigarren Hezkuntza. Natur Zientziak, Gasteiz, Eusko jaurlaritzaren Argitalpen Zerbitzu Nagusia.

HUTEAU,M. (1985): Les conceptions cognitives de la personnalité. Paris, P.U.F..

HUTEAU,M. (1987): Style cognitive \& Personnalité. La Dépendence-

Independence à l'égard du champ, Presses Universitaires de Lille.

INHELDER B., CELLÉRIER G. et al. (1992): Le cheminement des découvertes de l'enfant. Paris, Delachaux et Niestlé.

INHELDER y PIAGET (1955): De la logique de l'enfant à la logique de l'adolescence. Paris, P.U.F. Traducción al español: M.T. Cevasco: De la lógica del niño a la lógica del adolescente. Buenos Aires, Paidos, 1972.

KAGAN,J.; MOSS,H.A. y Sigel,I.E. (1973): Psychological signifiance of styles of conceptualisation. Wright,J.C. y Kagan,J., Basic Cognitive Processes in Children, Chicago and London, The University of Chicago Press, 73-112 o.

KAMII,C. (1981): Application of Piaget's theory to education, New Directions in Piagetian Theory and Practice, Edited by Irving E. Sigel;David M. Brodzinsky y Robert M. Golinkoff, L.E.A., Hillsdale, New Jersey. 231-265 o.

KAMII,C. y DE VRIES,R. (1985): La teoría de Piaget y la educación preescolar. Madrid, Visor libros.

KARPLUS (1981): Education and Formal thought- A modest Proposal, New Directions in Piagetian Theory and Practice, Edited by Irving E. Sigel; David M. Brodzinsky eta Robert M. Golinkoff, L.E.A., Hillsdale, New Jersey, 285-314.

KLEIN,G.S. (1970): Perception, Motives and Personality, New York, A. Knopf. 
KOGAN,N. (1976): Cognitive Styles in Infancy and Early Chilhood, Hillsdale, New Jersey, Lawrence Erlbaum Associates, Publishers.

LANGFORD,P. (1990): El desarrollo del pensamiento conceptual en la escuela secundaria. Barcelona, Paidos.

LEFEBVRE,G. y PORGE J.F. (1966): La médecine égyptienne. Taton,R., La Science Antique et Médiévale, Paris, P.U.F., 50-71.

LONGEOT,F. (1969): Psychologie différentielle et théorie opératoire de l'intelligence. Paris, Dunod.

LONGEOT,F. (1978): Les stades opératoires de Piaget et les facteurs de l'intelligence, Presses Universitaires de Grenoble.

LOPEZ RUPEREZ,F. y PALACIOS GOMEZ,C.(1988): La exigencia cognitiva en Física básica. Madrid, Centro de Publicaciones, C.I.D.E..

MORENO,M. (1983): La pedagogía operatoria. Barcelona, Laia.

MORENO,M.: Teoría del movimiento (bideoa), Barcelona, I.M.P.A.E.

MORENO,M. y SASTRE,G.(1987): Aprendizaje y desarrollo intelectual. Barcelona, Gedisa.

NEIMARK,E.D. (1981): Confounding with Cognitive Style Factors: An Artifact Explanation for the Apparent Nonuniversal Incidence of Formal Operations, Sigel,I.E; Brodzinsky,D.M y Golinkoff,R.M., New Directions in Piagetian Theory and Practice, Hillsdale, New Jersey, L.E.A., 177-189.

NOVAK,J.D. (1977): A theory of Education. Cornell, Cornell University Press. Traducción al español: Teoría y práctica de la educación. Madrid, Alianza, 1982.

OSBORNE,R. y FREYBERG P.(1985): Learning in Science. The implications of Children's science. Nueva Zelanda, Heinemann Publishers. Traducción al español: El aprendizaje de las Ciencias. Implicaciones de la ciencia de los alumnos. Madrid, Narcea, 1991.

PALACIOS,J. : Reflexividad-impulsividad, Infancia y aprendizaje, 17, 29-69, 1982.

PALACIOS,J. y CARRETERO,M.: Implicaciones educativas de los estilos cognitivos, Infancia y aprendizaje, 18, 86-106, 1982.

PASCUAL-LEONE,J. (1978): La teoría de los operadores constructivos. J.A. Delval (Comp.), Lecturas de Psicología del niño, Vol.I. Madrid, Alianza, 208-227 o. 
PIAGET,J. (1941): Le mecanisme du dévelopement mental et les lois du groupement des operations. Esquisse d'une théorie opératoire de l'intelligence. Archives de Psychologie, 28, 215-285. Traducción al español: J.A.Delval: El mecanismo del desarrollo mental. Madrid, Ed. Nacional, 1976.

PIAGET,J.(1949): Introduction à l'épistemologie génétique. Paris, P.U.F..(1/ La pensée mathématique, 2/ La pensée physique).

PIAGET,J. (1967): Biologie et connaissance. Paris, Gallimard.

PIAGET,J. (1970): Psychologie et épistémologie. Paris, Denöel, Bibliothèque "Médiations". Traducción española: Psicología y epistemología. Barcelona, Ariel, 1971.

PIAGET,J. (1970): The science of education and the psychology of the child. New York, Orion Press.

PIAGET,J. (1972): Problèmes de Psychologie génétique. Paris, Denoël-Gonthier. Traducción al español: Problemas de psicología genética. Barcelona, Ariel, 1980.

PIAGET,J.(1975): Problemas de epistemología genética, Barcelona, Ariel.

PIAGET,J. (1978): El punto de vista de Piaget. Delval,J. (Comp.), Lecturas de Psicología del niño, Vol.I, Madrid, Alianza, 166-185 o.

PIAGET,J. (1979): L'Epistémologie génétique. Paris, P.U.F. Que sais-je?.

PIAGET,J. (1977): La naissance de l'intelligence chez l'enfant. Suisse, Delachaux \& Niestlé.

PIAGET,J. y BETH,E.W.(1961): Epistémologie mathématique et psychologie. Paris, P.U.F.

PIAGET,J. y GARCIA, R. (1982): Psicogénesis e historia de la ciencia. Mexico, Ed. S.XXI.

PIAGET,J. (1984): La representación del mundo en el niño. Madrid, Morata.

POZO,J.I.(1987,a): La historia se repite: las concepciones espontáneas sobre el movimiento y la gravedad, Infancia y Aprendizaje, $n^{\circ} 38,69-87$.

POZO.J.I. (1987,b): Aprendizaje de la ciencia y pensamiento causal. Madrid, Ed. Visor.

POZO J.I. y CARRETERO, M. : Del pensamiento formal a las concepciones espontáneas: ¿Qué cambia en la enseñanza de la ciencia?, Infancia y Aprendizaje, 38, 35-52, 1987. 
POZO,J.I.: La historia se repite: Las concepciones espontáneas sobre el movimiento y la gravedad, Infancia y aprendizaje, 38, 69-87, 1987.

POZO,J.I. (1989): Teorías cognitivas del aprendizaje. Madrid, Morata.

POZO,J.I.; GOMEZ CRESPO M.A.; LIMON,M. y SANZ SERRANO,A. (1991): Procesos cognitivos en la comprensión de la ciencia: las ideas de los adolescentes sobre la química. Madrid, C.I.D.E..

ROMAN PEREZ, M. (1988): Inteligencia y potencial de aprendizaje. Madrid, Ed. Cincel.

SIEGEL,L. y BRAINERD,Ch.(1983): Alternativas a Piaget. Madrid, Pirámide.

SMOCK (1981): Constructivism and Educational Practices, New Directions in Piagetian Theory and Practice, Edited by Irving E. Sigel; David M. Brodzinsky y Robert M. Golinkoff, Hillsdale, New Jersey, L.E.A., 51-69 o.

SOLER E.; ALVAREZ L.; GARCIA A.; HERNANFDEZ J.; ORDOÑEZ J.J.; ALBUERNE F. y CADRECHA M.A. (1992): Teoría y práctica del proceso de enseñanzaaprendizaje. Madrid, Narcea.

TIBERGHIEN,A.(1985): El desarrollo de las ideas mediante la enseñanza. Driver,R; Guesne,E. y Tiberghien, A., Ideas científicas en la infancia y la adolescencia, Madrid, Morata.

VYGOTSKII,L.S. (1987): Pensamiento y lenguaje. Buenos Aires, La Pléyade.

VYGOTSKII,L.S. (1978): Mind in society. The development of higher psychological process. Cambridge,Ma.: Harvard University Press. Traducción al español: S.Furio: El desarrollo de los procesos psicológicos superiores, Barcelona, Crítica, 1979.

WITKIN H.A. y GOODENOUGH D.R. (1981): Cognitive Styles: Essence and Origins, International Universities Press, Inc. Traducción al español: Pilar Sanchez López: Estilos cognitivos: Naturaleza y orígenes. Madrid, Ed. Pirámide, 1985.

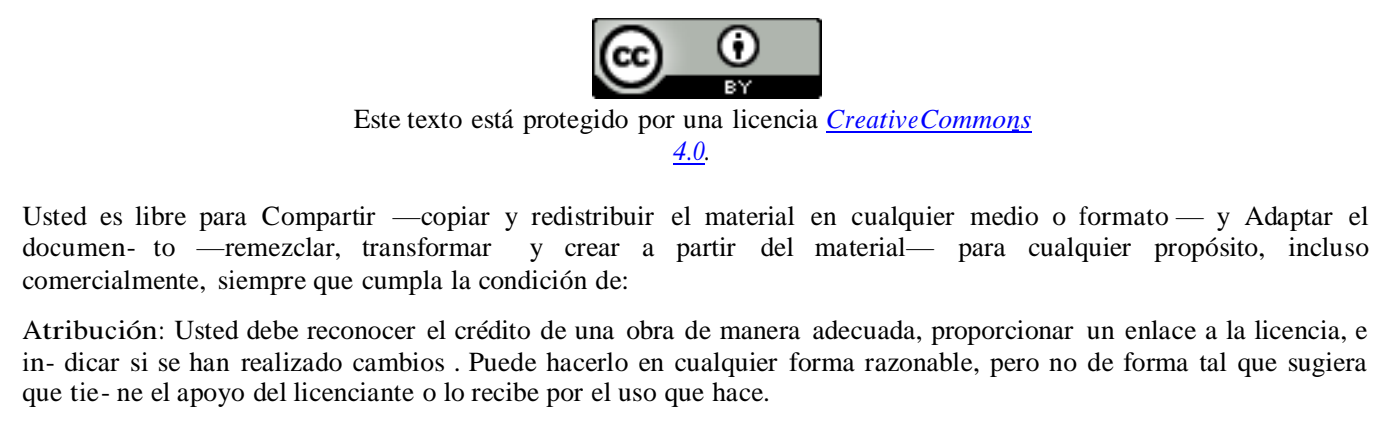

